Doug Geisler, Eva K. Grebel, and Dante Minniti, eds.

\title{
The Globular Clusters of M31: Keys to its Formation History
}

Kathy Perrett

Physics Dept., Queen's University, Kingston, ON K7L 3N6, Canada

Terry Bridges

Anglo-Australian Observatory, Epping, NSW, 1710 Australia

Dave Hanes

Physics Dept., Queen's University, Kingston, ON K7L 3N6, Canada

Mike Irwin

Institute of Astronomy, Cambridge University, Cambridge CB3 OHA, England, UK

Dave Carter

Astrophysics Research Institute, Liverpool JMU, Twelve Quays House, Egerton Wharf, Birkenhead, CH41 1LD, England, UK

Jean Brodie

Lick Observatory, University of California at Santa Cruz, Santa Cruz, CA 95064, USA

John Huchra

Harvard-Smithsonian Center for Astrophysics, 60 Garden Street, Cambridge, MA 02138, USA

Fred Watson

Anglo-Australian Observatory, Coonabarabran, NSW, 2357 Australia

\begin{abstract}
With the ultimate goal of distinguishing between various models describing the formation of galaxy halos (e.g., radial collapse, chaotic mergers), we present the results of a spectroscopic study of the globular cluster system of M31. We have obtained deep, intermediateresolution spectra for several hundred of the M31 globular clusters using the WYFFOS fibre-fed spectrograph at the William Herschel Telescope. These observations have yielded precise radial velocities and metallicities for over 200 members of the M31 globular cluster population, the vast majority of which represent new data or significant improvements over pre-existing data.
\end{abstract}




\section{Introduction}

In recent years, a number of different models have been proposed to explain the formation of galaxies and their globular cluster systems. An early model by Eggen, Lynden-Bell and Sandage (1962) called for a rapid, radial collapse of material to form a galaxy like the Milky Way. If the enrichment timescale is shorter than the collapse time, one would expect to find metallicity gradients in the halo. The lack of a significant abundance gradient in the outer Galactic halo prompted Searle and Zinn (1978) to propose that the Galaxy instead formed from chaotic accretion of dwarf galaxy sized lumps. Indeed, the presence of the Sagittarius dSph in the Milky Way is evidence that such mergers may be ongoing (Ibata et al. 1995). Many galaxies have shown signs of bimodality in their globular cluster (GC) colour/metallicity distributions, evidence that these galaxies contain multiple cluster populations and have experienced a somewhat more complex formation history. Other mechanisms have been proposed to explain the observed properties of globular cluster systems (GCSs), such as galaxy-galaxy interactions or gaseous mergers (e.g. Ashman and Zepf 1992), episodic in situ formation (Forbes et al. 1997), or the stripping and accretion of globulars within galaxy clusters (Côté et al. 1998).

Persuasive observational evidence to allow us to distinguish between these various models is still lacking. In order to address this problem, we undertook a spectroscopic study of the globular cluster system of M31. Globular clusters are well-suited for probing the dynamical and chemical enrichment histories of galaxies as well as for investigating its underlying mass distribution. M31 provides the ideal target due to its proximity and comparatively large globular cluster population, which was suffering from a surprising lack of published spectroscopic observations.

\section{The Data}

The data for this study were obtained at the William Herschel $4.2 \mathrm{~m}$ telescope (WHT) in La Palma, Canary Islands using the WYFFOS fibre-fed spectrograph (Bridges 1998). An overall spectral coverage of $\sim 3700-5600 \AA$ was obtained at intermediate resolution using two gratings to include absorption features such as the CH G-band, the Mgb triplet and Fe lines. Six fibre configurations were selected to target a total of 288 globular cluster candidates in $1^{\circ}$ fields over the full extent of the major axis of M31. Minor axis fields were not observed during this run due to poor weather and a shortage of time.

Radial velocities and metallicities were obtained for $\gtrsim 200$ cluster targets in the sample, the positions of which are shown in Fig. 1. The majority (109) of these clusters had no previously-published velocities and a large fraction of the remainder represent significant improvements over pre-existing data. Our adopted velocity uncertainty is $\pm 12 \mathrm{~km} \mathrm{~s}^{-1}$ and our median metallicity error is \pm 0.3 dex.

We merged our WYFFOS results with the velocities and $[\mathrm{Fe} / \mathrm{H}]$ values from other sources (Barmby et al. 2000; Dubath and Grillmair 1997; Federici et al. 1993; Huchra et al. 1991; Peterson 1989) to produce the best available 


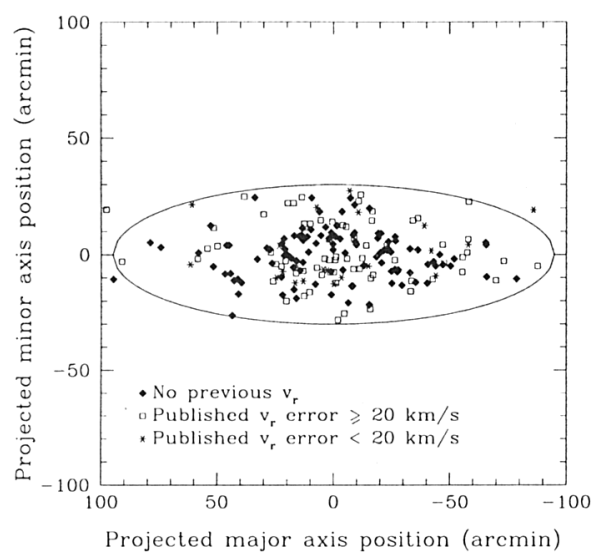

Figure 1. Positions of the $202 \mathrm{M} 31$ GCs for which radial velocities were obtained from the WYFFOS sample.

sample of spectroscopic data for subsequent analysis of the M31 globular cluster system (Perrett et al. in preparation).

\section{The GC Metallicity Distribution, Kinematics and Mass}

A KMM mixture modeling analysis (Ashman et al. 1994) reveals that the M31 GCS metallicity distribution is better represented by a bimodal distribution than by a single Gaussian at a high confidence level $(z 97 \%)$. The metal-poor and metal-rich cluster distributions have mean metallicities of $[\mathrm{Fe} / \mathrm{H}]=-1.44$ dex and -0.50 dex, respectively, and are shown in Fig. 2. The peak of M31's metal-poor cluster distribution lies at a higher abundance than its Milky Way counterpart at $[\mathrm{Fe} / \mathrm{H}]=-1.6 \operatorname{dex}($ Zinn 1985; Harris 1996).

Separating the M31 globular cluster population into metallicity sub-samples based on the KMM results, we find that the spatial distribution of metal-rich clusters is distinctly more centrally-concentrated. The metal-poor GCs demonstrate a gradient towards decreasing metallicity out to a galactocentric radius of $\sim 60^{\prime}$, confirming earlier results by Huchra et al. (1991). The metal-rich population is sparse but there is no evidence of an abundance gradient over its radial extent.

Interestingly, both the metal-rich and metal-poor cluster populations exhibit significant rotation (Fig. 3). We determine a rotation amplitude of $v_{\text {rot }}=160 \pm 19 \mathrm{~km} \mathrm{~s}^{-1}$ for the metal-rich population. This value is somewhat larger than the metal-poor amplitude of $v_{\text {rot }}=131 \pm 13 \mathrm{~km} \mathrm{~s}^{-1}$, although these values are not inconsistent within their formal errors.

The kinematics of the M31 GCS also provide us with a dynamical probe of the galaxy's mass distribution. We used the projected mass estimator (PME) to constrain the underlying mass of the system (Heisler et al. 1985). For isotropic orbits within an extended mass distribution, we estimate a mass of $\mathrm{M}_{\mathrm{M} 31}=$ $(4.1 \pm 0.1) \times 10^{11} \mathrm{M}_{\odot}$ using 319 cluster velocities out to a radius of $\sim 120^{\prime}$ 


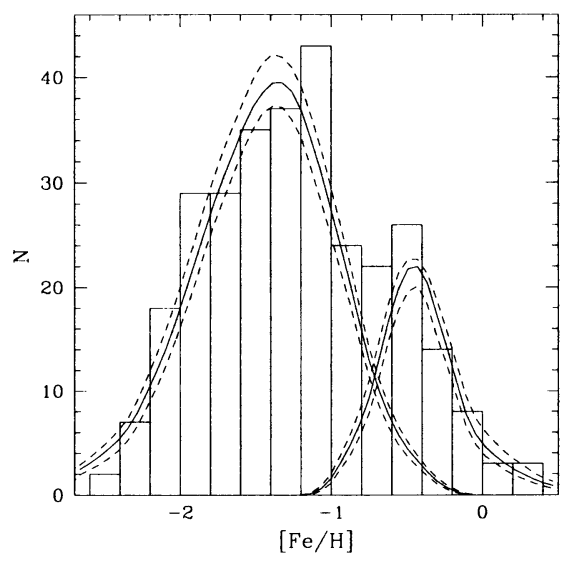

Figure 2. Adaptive kernel fits to the M31 metal-poor and metal-rich GC populations (solid lines), separated based on KMM mixture modeling results. The dashed lines represent bootstrap errors and provide the $5 \%$ and $95 \%$ confidence intervals.

from the galaxy center (which corresponds to a radius of $27 \mathrm{kpc}$ at a distance of $770 \mathrm{kpc}$ ). This result is consistent with previous mass estimates obtained out to similar radii as summarized in Table 6 of Evans and Wilkinson (2000).

\section{Future Work}

Preliminary substructure analysis of the available data suggests the presence of M31 globular clusters grouped in position/velocity/metallicity parameter space. Ashman and Bird (1993) and Saito and Iye (2000) have found similar signs of clustering in the M31 GCS. If these groups prove to be robust, they may represent the remnants of recent merger or accretion events as proposed by Searle and Zinn (1978).

We are currently completing the analysis of $\sim 200$ additional M31 GC spectra obtained using the MOS/ARGUS integral field spectrograph at the CanadaFrance-Hawaii Telescope, data which extend further from the major axis than the WYFFOS sample. A full kinematic and enrichment study of the GCs in M31 and other galaxies will provide us with essential insights into the formation and chemical evolution of globular cluster systems and their parent galaxies.

\section{References}

Ashman, K. M., \& Bird, C. M. 1993, AJ, 106, 2281

Ashman, K. M., Bird, C. M., \& Zepf, S. E. 1994, AJ, 108, 2348

Ashman, K. M., \& Zepf, S. E. 1992, ApJ, 384, 50

Barmby, P., Huchra, J. P., Brodie, J. P., Forbes, D. A., Schroder, L. L., \& Grillmair, C. J. 2000, AJ, 119, 727 


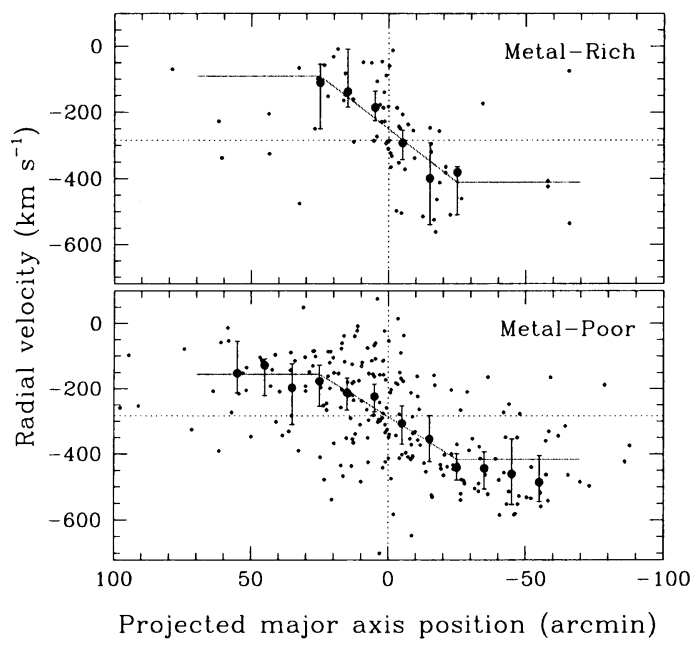

Figure 3. Rotation curves for the metal-rich and metal-poor subpopulations of the M31 GCS. The large filled circles represent robust biweight estimates of the mean in $10^{\prime}$ radial bins and the associated error bars show their $90 \%$ confidence interval (Beers et al. 1990). The solid line gives the best fit to the data assuming solid-body rotation in the inner $25^{\prime}$ and flattening beyond.

Beers, T. C., Flynn, K., \& Gebhardt, K. 1990, AJ, 100, 32

Côté, P., Marzke, R. O., \& West, M. J. 1998, ApJ, 501, 554

Dubath, P., \& Meylan 1997 A\&A, 321, 379

Eggen, O. J., Lynden-Bell, D., \& Sandage, A. R. 1962, ApJ, 136, 748

Evans, N. W., \& Wilkinson, M.I. 2000, MNRAS, 316, 929

Federici, L., Bònoli, F., Ciotti, L., Fusi Pecci, F., Marano, B., Lipovetsky, V. A., Neizvestny, S. I., \& Spassova, N. 1993, A\&A, 274, 87

Forbes, D. A., Brodie, J. P., \& Grillmair, C. J. 1997, AJ, 113, 1652

Harris, W. E. 1996, AJ, 112, 1487 (McMaster catalogue, revised 1999).

Heisler, J., Tremaine, S., \& Bahcall, J. N. 1985, ApJ, 298, 8

Huchra, J. P., Brodie, J. P., \& Kent, S. M. 1991, ApJ, 370, 495

Ibata, R. A., Gilmore, G., \& Irwin, M. J. 1995, MNRAS, 277, 781

Peterson, R. 1989, in Dynamics of Dense Stellar Systems, ed. D. Merritt (Cambridge: Cambridge Univ. Press), 161

Saito, Y. and Iye, M. 2000, ApJ, 535, L95

Searle, L., \& Zinn, R. 1978, ApJ, 225, 357

Zinn, R. 1985, ApJ, 293, 424 


\section{Discussion}

P. Goodfrooij: Your spectra looked good enough to actually measure relative line strength ratios for many clusters. Can you say anything about $\alpha / F e$-peak element ratios for the metal-rich globular clusters relative to the metal-poor ones?

K. Perrett: We do have good $\mathrm{Mg}$ and Fe line strengths for many of the clusters in our sample, and will be completing this type of analysis shortly. Unfortunately, I can't comment on the $\alpha / F e$ ratios for the metallicity sub-populations yet.

M. Kissler-Patig: Did you check whether your spectroscopic sample is biased with respect to metallicity? i.e is your sample representative of the total population? As a proposed test: plot the spectroscopic sample color distribution vs. the color distribution of the total population.

K. Perrett: Excellent point! This is certainly a concern but we have not yet characterized any potential bias. Your suggestion is a good one and we will test for sample bias.

M. Drinkwater: Have you considered using the KMM algorithm to search for substructure in more than just one parameter simultaneously? For instance, you might get a more significant partition of the metal-rich/metal-poor subsets by including the two sky coordinates as well (rotated if necessary to line up with the galaxy's axes).

K. Perrett: No, we use KMM only to search for multi-modality in the metallicity distribution. The KMM algorithm presumes an underlying Gaussian parent population and has been shown to be adequate for testing the cluster metallicity distribution (Ashman \& Bird 1993). It is not clear to me how the additional parameters would improve the partition between the metal-rich and metal-poor subsets. We use other techniques to search for substructure and correlations in position-velocity-metallicity parameter space.

B. Harris: Could you comment on the precision of your new metallicity measurements compared with previous studies? What happens to the $[\mathrm{Fe} / \mathrm{H}]$ histogram if you select the highest-precision objects - does it become more clearly bimodal?

K. Perrett: Our metallicity uncertainties are typically of order $0.5-0.8 \mathrm{dex}$. The $[\mathrm{Fe} / \mathrm{H}]$ distribution shape does not seem to change substantially with the exclusion of the lower precision results.

D. Forbes: Can you comment on the velocity dispersion properties of the two populations?

K. Perrett: It appears as though the velocity dispersion for the metal-poor population is roughly $150 \mathrm{~km} / \mathrm{s}$ at small $\mathrm{R}$, and decreases to $120 \mathrm{~km} / \mathrm{s}$ or so beyond $\mathrm{R}=25$ arcmin. The metal-rich cluster sample demonstrates a very flat dispersion profile at around $125 \mathrm{~km} / \mathrm{s}$ throughout the radial extent of the population. 